\title{
E-HRM COMO INTRAEMPREENDEDORISMO: IMPACTO NO DESEMPENHO E RESULTADOS ORGANIZACIONAIS
}

Geizi Cássia Bettin Do Amarante ${ }^{1}$ Anelise Rebelato Mozzato ${ }^{1}$

André Da Silva Pereira ${ }^{1}$

${ }^{1}$ Universidade de Passo Fundo 


\section{E-HRM COMO INTRAEMPREENDEDORISMO: IMPACTO NO DESEMPENHO E RESULTADOS ORGANIZACIONAIS}

Resumo: A velocidade da tecnologia da informação (TI) e inovações pressionam as organizações, as quais necessitam se mobilizarem. A área de gestão de recursos humanos (GRH) também sofre esse impacto, a emergência para se tornar mais eficiente, produtiva, inovadora e capaz de apoiar os objetivos estratégicos, assim, impelida a trabalhar com a gestão eletrônica de recursos humanos, a e-HRM. Dada a emergência do tema, esta revisão sistemática de literatura analisa as práticas de e-HRM, relacionando com o desempenho e resultados organizacionais, na perspectiva do intraempreendedorismo. Por meio da apresentação dos resultados, essa investigação resulta na confirmação de que a e-HRM se apresenta como uma alternativa eficiente, produtiva, inovadora e capaz de apoiar os objetivos estratégicos de uma organização, através de uma gestão estratégica de recursos humanos (GERH). Dessa forma, a e-HRM constitui-se como intraempreendedorismo para a GERH, fazendo-se necessária e cada vez mais oportuna frente a exigências da quarta revolução industrial.

Palavras-chave: e-HRM, Desempenho e resultados Organizacionais. Intraempreendedorismo. Revisão sistemática de literatura.

\section{Introdução}

Nos últimos anos, em crescimento exponencial, os sistemas de informação constituem um dos principais campos de estudo na organização, sendo possível detectar a influência da tecnologia da informação (TI) nos resultados organizacionais (ALMAZÁN; TOVAR; QUINTERO, 2017; IQBAL et al., 2019). Os resultados obtidos por Almazán, Tovar e Quintero (2017) permitiram deduzir que as organizações que prestam mais atenção à melhoria da qualidade do sistema, da informação e dos sistemas de informação, satisfazem seus resultados organizacionais. E ainda, alguns resultados fornecem evidências que a gestão eletrônica de recursos humanos, do inglês e-HRM, aprimora a confiança organizacional e os resultados de produtividade (IQBAL; AHMAD; ALLEN, 2019). Inclusive, Ash (2007) verificou que o tema se encontra entre os que influenciam os resultados organizacionais.

Considerando os desafios na pesquisa de gestão de recursos humanos (GRH) aliado ao crescimento tecnológico, surgiram as discussões sobre e-HRM e seu papel nas organizações, a partir dos estudos centrados em recursos humanos (RH) e TI (BONDAROUK; RUEL, 2009). Portanto, a GRH é percebida como um termo abrangente e que objetiva gerar valor para as organizações, fortalecendo o seu pressuposto de atuar estrategicamente com a finalidade de reduzir custos e tornar-se mais eficiente, flexível e orientada para o cliente, através do uso da TI, assim seja, o e-HRM.

As práticas de e-HRM advém da abordagem da gestão do conhecimento (FERRARESI, 2014), a qual trata o conhecimento como recurso estratégico, que por sua vez é embasado na teoria da organização baseada em recursos (resource based view of the firm - RBV), proposta por Penrose (1959). A RBV denomina o conhecimento como um recurso organizacional pode incluir qualquer ativo específico, tangível ou intangível, ligado de maneira semipermanente a uma organização, e é necessário, estratégias para alavancar esses recursos únicos (WERNERFELT, 1984; BARNEY, 1991; PETERAF, 1993; GRANT, 1996).

Nesse contexto, entende-se que as práticas de e-HRM se correlacionam com as práticas

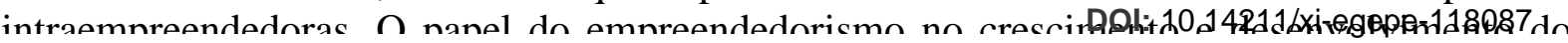


ambiente organizacional é um fato consolidado. Frente às inovações constantes que trazem pressões nesse cenário emergente, corrobora-se com Pereira e Hashimoto (2014) quanto ao fato de que o empreendedorismo envolve o reconhecimento da oportunidade para criar algo (não necessariamente um produto ou um serviço), a inspiração, fundamental para a criação de algo novo, que as pessoas atribuirão algum valor. Diante do reconhecimento da importância do empreendedorismo, surgem diferentes formas de empreender, dentre elas, o intraempreendedorismo.

Empreendedores e intraempreendedores são atores sociais que ao desenvolverem suas competências atribuem potencialidades ao mercado (NASSIF; ANDREASSI; SIMÕES, 2011). Isto posto, intraempreendedor é aquele que transforma uma ideia em realidade dentro de uma organização, podendo ser ou não a pessoa a apresentar a primeira ideia, mas que não mede esforços para fazer o que é preciso ser feito para alcançar os melhores resultados (MUNHOZ; NASSIF, 2012). O termo intraempreendedorismo surgiu nos anos 70, com Gifford Pinchot, visando caracterizar as ações das organizações no sentido de criar um ambiente de incentivo à inovação, à aceitação de riscos e à criatividade dentro das organizações (HASHIMOTO, 2012).

O intraempreendedorismo é visto como uma das formas de empreendedorismo corporativo em organizações existentes, a partir de um processo no qual os trabalhadores buscam oportunidades, independentes dos recursos que controlam (NASSIF; ANDREASSI; SIMÕES, 2011). As práticas intraempreendedoras, segundo Haschimoto (2012) são ações desenvolvidas pelas organizações e pelos seus trabalhadores no sentido de facilitar que o fenômeno do empreendedorismo corporativo aflore dentro dessas organizações. Assim sendo, os gestores devem ter em mente a importância de fatores como a orientação para o mercado, a flexibilidade e satisfação no trabalho no planejamento de estratégias e práticas para avançar o empreendedorismo corporativo.

Dentro desse contexto de intraempreendedorismo, Lapolli e Gomes (2017) destacam algumas práticas: prática de reconhecimento dos intraempreendedores; práticas para fomentar a criatividade e a inovação; prática de comunicação; prática de liberdade de ação; prática de tolerância a erros. As principais práticas utilizadas estão ligadas a maior autonomia dos trabalhadores, recompensas financeiras e não pecuniárias, permanência limitada nos cargos e a tolerância a erros. Porém, as dificuldades nesse processo, sendo o excesso de burocracia e o apego às normas e regulamentos os principais entraves ao intraempreendedorismo (LAPOLLI; GOMES, 2017).

Nesse sentido, considerando as mudanças emergentes e as inovações tecnológicas no campo organizacional, o objetivo deste trabalho é apresentar uma revisão sistemática de literatura sobre as práticas de e-HRM e seus impactos organizacionais numa perspectiva de intraempreendedorismo. Justifica-se a realização desse estudo tendo em vista o que argumentaram Bondarouk e Ruel (2009) sobre questões importantes e desafiadoras para o futuro da HRM e, ainda, conforme ressaltado por Iqbal et al. (2019) ao adotar as práticas de eHRM, pode-se agregar valor às organizações e, assim, a área de RH pode contribuir para a obtenção de resultados estratégicos. Aliado a isso, a quarta revolução industrial, que faz com que as organizações necessitem se modernizar para atender a demanda e as exigências dos consumidores modernos (COLTRE; MARTINS, 2019).

As revisões de literaturas, bem como pontua Yin (2016), tem sido utilizada por pesquisadores no âmbito científico com o objetivo de reunir conhecimento sobre um determinado tema, analisando o seu histórico e progresso no decorrer do tempo, bem como suas controversas. Assim, desenvolve-se esta revisão de literatura sobre e-HRM possibilitando que pesquisadores interessados no tema possam conhecer a literatura existente sobre a temática que desejam pesquisar, garantindo maior segurança para discorrer sobre, assim como para apontar

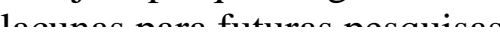
DOI: $10.14211 /$ xi-egepe-118087 
Isto posto, esta revisão sistemática de literatura comtempla abordagens teóricas que contextualizam as práticas de e-HRM e suas implicações nas organizações. Primeiramente, buscou-se apresentar uma revisão de literatura sobre o surgimento e o avanço das práticas de eHRM, simultaneamente com o avanço e as inovações tecnológicas. Na sequência discorre-se sobre os resultados encontrados com a revisão sistemática da literatura e, por fim, as considerações finais, salientando as implicações para pesquisas futuras.

\section{Abordagem teórica}

\subsection{E-HRM como intraempreendedorismo}

A preocupação com o impacto das práticas de HRM na percepção de desempenho organizacional (DELANEY; HUSELID, 1996) é pesquisado há décadas, entretanto, como surgem novos fatores, a exemplo da diversidade (ASH, 2007) e da tecnologia (ALMAZÁN; TOVAR; QUINTERO, 2017), fazem com que a pesquisa, além de estratégica, torne-se constante e emergente. Porém, a maioria dos estudos são voltados para desempenho organizacional, enquanto, poucos são especificamente, sobre resultados organizacionais. Para tanto, faz-se necessário a discriminação entre o que é desempenho e resultado organizacional.

A gestão de resultados nas organizações surgiu em 1954 com Peter Drucker, ao definir a gestão por objetivos (management by objectives - MBO). Assim sendo, uma gestão voltada aos resultados organizacionais é um modelo que enfatiza a importância da definição de objetivos e da avaliação isolada de cada área, propondo desafios aos gestores (DRUCKER, 2012). Portanto, exige bastante comprometimento da equipe. Gestão por objetivos é uma ferramenta para fazer a integração do propósito (ganho, crescimento e desenvolvimento) com as necessidades individuais do gestor (renda, pagamento, melhoria e afins) (ISLAMI; MULOLLI; MUSTAFA, 2018).

Por outro lado, desempenho organizacional avalia resultados através de indicadores, para isso, surgiram os modelos de desempenho organizacional, comunicar objetivos e definir claramente as metas que devem ser realizadas pelos trabalhadores, resulta em alcançá-los, trabalhando com satisfação pelos trabalhadores (ISLAMI; MULOLLI; MUSTAFA, 2018). Destaca-se as duas medidas de desempenho organizacional que têm sido adotadas pela comunidade acadêmica: objetivas e subjetivas. As medidas objetivas incluem faturamento, número de trabalhadores, resultado operacional, entre outras. Já as medidas subjetivas são coletadas pela opinião do sujeito pesquisado. Venkatraman e Ramanujam (1987) estudaram a convergência destes dois métodos, indicando que os dois tipos de medida se relacionam e podem ser usados de acordo com a necessidade e conveniência do pesquisador.

Entre indicadores, modelos e sistemas, Becker e Huselid (1998) mencionam os sistemas de trabalho de alto desempenho (High Performance WorkSystems - HPWS) quando há sinergia entre o conjunto do sistema de gestão de pessoas e os objetivos estratégicos. Essa coerência é importante para entender a relação entre a HRM e o desempenho organizacional. As organizações estabelecem indicadores para avaliar os resultados do programa e analisar se foi uma experiência de impacto positivo. Portanto, este estudo tem por objetivo verificar se as práticas de e-HRM podem estrategicamente impactar no desempenho e nos resultados organizacionais.

A tomada de decisão de implementação de e-HRM, é delicada, o gerenciamento o de RH precisa de atenção durante o processo (MASUM, KABIR, CHOUWDHURY, 2015). Fazse necessário explorar os determinantes que influenciam a decisão de adaptação para e-HRM entre organizações, havendo então oportunidades para intraempreendedores Nura e e Osman 
base em tecnologia e e-HRM voltados para a retenção de trabalhadores, enfatizando o papel dos trabalhadores para o desempenho organizacional e para adaptação do e-HRM.

Tais iniciativas envolvem o intraempreendedorismo, como já mencionado, termo cunhado por Gifford Pinchot III no final da década de 197, o qual diz respeito a tomada de diferentes ações nas organizações, buscando criar um ambiente que incentive a inovação e a criatividade dentro delas. Pinchot (1989) constatou que há trabalhadores com características e comportamentos empreendedores, mas que não tem a intenção de empreender fora das organizações em que trabalham. Esses que o autor chama de intraempreendedores. Profissionais com esse perfil nas organizações tem condições de assumir a responsabilidade pela criação de inovações de qualquer espécie, constituindo-se em importantes impulsores de inovação e renovação estratégica.

Na última década, como referem Baruah e Ward (2014), nesse ritmo acelerado de constantes mudanças econômicas e organizacionais, o comportamento empreendedor tornouse uma competência necessária aos profissionais que atuam em diferentes organizações. Assim, o conceito atual de empreendedor inclui também os trabalhadores que desenvolvem e realizam atividades internas em favor de melhorias em processos e de produtos, na busca de melhores desempenho e resultados organizacionais.

\subsection{Desempenho e resultados organizacionais}

Na busca pela validação dos resultados de e-HRM, Bayarçelik e Findikli (2015) através de entrevistas aprofundadas com o especialista em RH de uma indústria de serviços mostrou que o gerenciamento do tempo, a fácil aquisição e acesso a dados pessoais e a redução dos custos de administração foram os principais motivadores para as aplicações de recursos humanos eletrônicos. O e-HRM reduziu os custos organizacionais, melhorou a comunicação entre o gerente e os trabalhadores, reduzindo o tempo de processamento para o uso de e-HR nas organizações. No entanto, em questões mais específicas, a exemplo do e-learning/etraining, pesquisas demonstram que os resultados não são muito eficazes para as organizações (BAYARÇELIK; FINDIKLI, 2015).

No que se refere a gestão estratégica de pessoas, Marler e Fisher (2013) denotam que é justamente esse o objetivo da e-HRM, tornar a função de HRM mais estratégica. Em relação as questões emergentes em teoria e pesquisa de e-HRM, Stone e Dulebohn (2013) apuraram que a tecnologia teve um impacto dramático nos processos e práticas HRM. A TI ajudou a modificar muitos processos de HRM, incluindo planejamento de $\mathrm{RH}$, recrutamento, seleção, gerenciamento de desempenho, fluxo de trabalho e remuneração. Esses novos sistemas permitiram que os profissionais de HRM prestassem um serviço melhor a todas as partes interessadas (por exemplo, candidatos, trabalhadores, gerentes) e reduzissem a carga administrativa no campo (STONE; DULEBOHN, 2013).

Procurando verificar a importância da aplicação do MBO, como método de avaliação de desempenho, voltado a efetividade dos trabalhadores, Islami, Mulolli e Mustafa (2018) sustentam que o método MBO deve ser usado como um método de avaliação de desempenho à medida que a eficácia dos trabalhadores é aumentada. Também resultou que a avaliação do desempenho dos trabalhadores individuais e uma definição clara dos resultados são os parâmetros mais importantes de todas as outras atividades do método. Também resultou que a avaliação do desempenho dos trabalhadores individuais e uma definição clara dos resultados são os parâmetros mais importantes de todas as outras atividades do método MBO no estudo 
para aumentar a eficácia dos trabalhadores na organização (ISLAMI; MULOLLI; MUSTAFA, 2018).

Ao analisar a relação entre práticas de HRM e resultados organizacionais, Esteves e Caetano (2010), evidenciaram que o êxito organizacional está relacionado com a forma como as organizações gerem as pessoas que nelas trabalham (ESTEVES; CAETANO, 2010). Inclusive, Esteves e Caetano (2010) sugerem também que é conveniente pesquisar a possível existência de HRM diferenciadas, para grupos funcionais distintos. Neste caso, pode-se indicar as categorizações de e-HRM, delimitadas por IQBAL et al. (2019): e-HRM operacional, relacional e transformacional.

Em uma aplicação da teoria institucional à e-HRM e sistemas de informações estratégicas de maneira mais geral, em um ambiente subsidiário de uma corporação multinacional, Heikkilä (2013) pesquisou fatores institucionais de um país nas práticas estratégicas de e-HRM em subsidiárias estrangeiras. Os resultados ilustraram como as pressões institucionais criam tanto consequências transformacionais negativas para as subsidiárias, e que as respostas das subsidiárias a essas pressões podem afetar substancialmente a capacidade de atingir seu potencial estratégico, impactando até mesmo no desempenho dos trabalhadores e da organização.

\section{Metodologia}

Para a realização dessa pesquisa foi utilizado procedimento qualitativo de revisão sistemática da literatura, uma metodologia eficaz para integrar as informações de um conjunto de trabalhos realizados separadamente, ou seja, investigar o atual estado da arte, como afirmam Martins et al. (2018). A revisão sistemática gera resultados com materiais críticos e de qualidade, como também ganha importância atual em trabalhos científicos de diferentes áreas pesquisadas (SAMPAIO; MANCINI, 2007; GUARNIERI, 2015; MARTINS et al., 2018; FILIPPI; GUARNIERI; CUNHA, 2019). Além da robustez teórica, possibilitam identificar novos temas e lacunas para estudos futuros.

Uma revisão sistemática, assim como outros tipos de revisões constituem-se numa forma de pesquisa que utiliza como fonte de dados a literatura sobre determinado tema (SAMPAIO; MANCINI, 2007). Neste estudo, a revisão é realizada de acordo com o protocolo de Cronin, Ryan e Coughlan (2008), que fora proposto baseado em modelos utilizados na medicina e utilizado por autores como Higgins e Green (2011). Assim, as diferentes etapas desta revisão sistemática baseada em Filippi, Guarnieri e Cunha (2019) e são detalhadas na Quadro 1. 


\section{Quadro 1 - Etapas do protocolo de revisão sistemática de literatura e-HRM}

\begin{tabular}{|l|l|l|}
\hline Etapas & Denominação & Desenvolvimento \\
\hline $1^{\mathbf{0}}$ & $\begin{array}{l}\text { formulação da questão de } \\
\text { pesquisa }\end{array}$ & $\begin{array}{l}\text { Considerando as mudanças emergentes e as inovações tecnológicas } \\
\text { no campo organizacional, sob a ótica do intraempreenderorismo, as } \\
\text { práticas de e-HRM impactam o desempenho e os resultados } \\
\text { organizacionais? }\end{array}$ \\
\hline $2^{\circ}$ & $\begin{array}{l}\text { conjunto de critérios de } \\
\text { inclusão e exclusão }\end{array}$ & $\begin{array}{l}\text { Os critérios de inclusão e exclusão utilizados nas buscas da literatura } \\
\text { existente obedeceram às seguintes delimitações para obter materiais: } \\
\text { (a) palavra-chave em inglês e-HRM com exceção para Science } \\
\text { Direct; (b) apenas artigos completos em periódicos (exclusão de } \\
\text { resumos, capítulos de livros, anais de eventos, editoriais, patentes } \\
\text { etc.); (c) período de publicação: livre; e (d) base de dados } \\
\text { internacionais: Science Direct, Taylor \& Francis, Emerald Insight e } \\
\text { Scopus, e base de dados nacionais: Scielo e Spell. }\end{array}$ \\
\hline $3^{\mathbf{0}}$ & $\begin{array}{l}\text { seleção e acesso da literatura } \\
\text { Artigos completos publicados nos periódicos selecionados de acordo } \\
\text { com os critérios de inclusão exclusão estabelecidos. Primeiramente } \\
\text { foi realizada uma filtragem com base nos títulos e resumos dos } \\
\text { trabalhos. }\end{array}$ \\
\hline $4^{\mathbf{0}}$ & $\begin{array}{l}\text { avaliação da qualidade da } \\
\text { literatura incluída na revisão }\end{array}$ & $\begin{array}{l}\text { A partir das buscas e dos critérios estabelecidos no protocolo, foram } \\
\text { encontrados 28 artigos. Para tanto, avaliou-se previamente através de } \\
\text { uma leitura dos resumos e documentos. }\end{array}$ \\
\hline $5^{\circ}$ & $\begin{array}{l}\text { (análise, síntese } \\
\text { disseminação dos resultados }\end{array}$ & $\begin{array}{l}\text { Os 28 artigos selecionados foram analisados rigorosamente. Optou- } \\
\text { se em elaborar tabelas, quadros e fluxos para melhor visualização e } \\
\text { análise dos resultados. }\end{array}$ \\
\hline
\end{tabular}

Fonte: Elaborado de acordo com Filippi, Guarnieri e Cunha (2019).

Vale ressaltar duas observações sobre o protocolo estabelecido para busca, na base Science Direct para não haver confusão do termo de busca, foi necessário incluir também human resource managent, pois a base retornava 47.436 resultados, ressurgindo muitos artigos que não faziam parte da área de GRH. A segunda observação é referente ao não estabelecimento de período, uma vez que, pretende-se mapear o tema de pesquisa, por ser relativamente novo, assim, sendo possível identificar precursores do tema e quando do seu surgimento. O retorno das buscas e os artigos selecionados estão contabilizados na Tabela 1.

Tabela 1 - Bases de dados e respectivo número de artigos encontrados

\begin{tabular}{|l|l|l|}
\hline BASE DE DADOS & $\begin{array}{l}\text { ARTIGOS ENCONTRADOS } \\
\text { NA BUSCA }\end{array}$ & AMOSTRA SELECIONADA \\
\hline SCIENCE DIRECT & 330 & 8 \\
\hline TAYLOR \& FRANCIS & 176 & 8 \\
\hline EMERALD INSIGHT & 142 & 5 \\
\hline SCOPUS & 40 & 6 \\
\hline SCIELO & 0 & 0 \\
\hline SPELL & 1 & 1 \\
\hline TOTAL & 689 & 28 \\
\hline
\end{tabular}

Fonte: Dados da pesquisa (2019)

Para a formação da amostra dos dados do estudo, a busca sistemática está relacionada na tabela 1. Nessa busca dos textos científicos, de acordo com os termos, foram inicialmente encontrados 689 artigos. Na sequência todos os textos foram analisados, cumprindo os critérios de inclusão e exclusão. assim, após a leitura e análise foram selecionamentos 28 artigos, os quais serão trabalhados na sequência. 


\section{Resultados e discussões da literatura}

Visando tornar a área de HRM mais estratégica as organizações buscam melhorar a capacidade e os processos por meio da implementação e do desenvolvimento de e-HRM (MARLER; FISHER, 2013), a partir do paradigma teórico da RBV, que tem sido extensivamente usado na literatura estratégica (MARLER; FISHER, 2013; IQBAL et al., 2019). Geralmente a HRM refere-se a um conjunto de atividades realizadas por uma organização para a utilização efetiva do capital humano (IQBAL et al., 2019). De acordo com Bayarçelik e Findikli (2015) essas atividades podem ser divididas em três categorias principais: atividades transacionais (derivadas do impacto operacional), atividades tradicionais (derivadas de impactos relacionais) e atividades transformacionais (derivadas do impacto transformacional).

Com a expansão da internet surgiram novas formas de HRM, e, com as novas possibilidades, obteve-se mudanças importantes, como por exemplo no recrutamento tradicional (GARCÍA-IZQUIERDO; RAMOS-VILLAGRASA; CASTAÑO, 2015). E com a pressão excepcional que HRM sofre para se tornar mais eficiente, produtiva, inovadora e capaz de apoiar os objetivos estratégicos, nasce uma nova abordagem de HRM, a gestão eletrônica de recursos humanos (e-HRM) (IQBAL et al., 2019). Paralelo ao desenvolvimento das TIs, a eHRM tornou-se um assunto dentro da literatura acadêmica em 1995 (STROHMEIER, 2007). A definição de Bayarçelik e Findikli, (2015) par e-HRM é de um método de implementação de várias estratégias, políticas e práticas de GRH dentro das organizações, meios de suporte consciente e direto e uso de canais de tecnologia baseados na web. O e-HRM também pode ser definido, de acordo Strohmeier (2007), como inserção de TI para apoiar e colocar em rede pelo menos dois atores individuais ou seu desempenho compartilhado de tarefas de $\mathrm{RH}$.

Existem algumas categorizações para e-HRM, contudo, as três principais agrupações são: operacional, relacional e transformacional (BAYARÇELIK; FINDIKLI, 2015; IQBAL et al., 2019). E ainda, Iqbal et al. (2019) ressaltam que a e-HRM não tem um papel específico no desenvolvimento da HRM, mas oferece um conjunto de abordagens para ela. As práticas operacionais de e-HRM estão voltadas ao papel administrativo (IQBAL et al., 2019) e melhoram a eficácia e eficiência da HRM, justamente pelas possibilidades de automação e suporte (STROHMEIER, 2007; BAYARÇELIK; FINDIKLI, 2015).

As práticas relacionais de e-HRM enfocam relacionamentos interpessoais (IQBAL et al., 2019). Visam melhorar as relações com os interessados e a prestação de serviços de e-HRM e estão preocupados com os negócios, apoiando processos por meio de treinamento, recrutamento e gerenciamento de desempenho (BAYARÇELIK; FINDIKLI, 2015). Como afirmam Bissola e Impertori (2013), constitui-se em ferramenta para gerenciar o relacionamento entre organizações e seus trabalhadores, tendo propósito duplo, como referem Iqbal et al. (2019): primeiro, melhoram a qualidade dos serviços de RH e, segundo, asseguram justiça processual e organizacional capacitando trabalhadores e gerentes. A utilização da eHRM como meio para aumentar o valor da função de RH, foi observada por Parry (2011). Para a autora, o uso potencial da e-HRM sugeriu que ela pode apoiar a função de RH, tornando-a mais eficiente, melhorando assim a prestação de serviços e adotando um papel mais importante na execução da estratégia de negócios da organização.

E ainda, a e-HRM transformacional diz respeito às práticas caráter estratégico (BISSOLA; IMPERATORI, 2013), para alinhar a atitude e o comportamento dos trabalhadores com a estratégia da organização gerentes (IQBAL et al., 2019). Além disso, significa desenvolvimento no apoio ao negócio e orientação estratégica da e-HRM, como a gestão do conhecimento e reorientação estratégica (BAYARÇELIK; FINDIKLI, 2015). A tecnologia eHRM é uma maneira de implementar estratégias, políticas e práticas de RH (YUSLIZA; RAMAYAH, 2012). A TI suporta a função de cumprir as necessidades de RH da organização

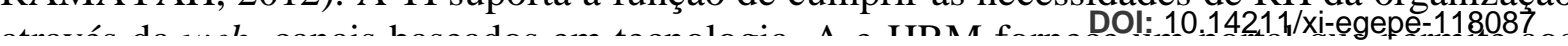


gerentes, trabalhadores e profissionais para visualizar, extrair ou alterar informações necessárias para o gerenciamento o RH da organização (YUSLIZA; RAMAYAH, 2012).

Algumas vantagens da e-HRM para uma organização internacional são destacadas por Burbach e Royle (2014), incluindo o acesso a todas as informações relacionadas a RH de um banco de dados central; alinhamento de todos os processos, políticas e procedimentos relacionados a $\mathrm{RH}$ em todo a multinacional; estabelecimento de uma variedade de métricas de RH; possibilidade de usar o autoatendimento de RH do trabalhador e do gerente; bem como, o uso de centros de serviços de RH. Os autores ainda sugerem que as vantagens da e-HRM podem ser evidenciadas nos níveis individual, operacional, relacional e transformacional.

Precursores dos debates sobre e-HRM, constando entre os principais pesquisadores, Ruel, Bondarouk e Looise (2004) destacaram a substituição das atividades de HRM presenciais por ferramentas de gestão baseadas na Web, no caso e-HRM. Ruel, Bondarouk e Van der Velde (2007) evidenciaram a expectativa otimista de que a tecnologia possibilitava assumindo que, de uma perspectiva técnica, as possibilidades de TI e HRM eram infinitas (). Logo após, Bondarouk e Ruel (2009; 2013), enfatizaram os desafios na era digital e deram continuidade ao debate ao aplicar a abordagem de capacidades organizacionais e por meio de métodos mistos de pesquisa, ressaltando que era necessário disseminar a importância da e-HRM por toda a organização, para então alcançar resultados estratégicos.

Desde o surgimento das práticas de e-HRM, a maioria dos estudos se conteve em organizações nacionais, portanto, Bondarouk, Schilling e Ruel (2016) testaram os fatores ambientais que influenciam a adoção do e-HRM, incluindo a decisão de adotar um certo sistema de e-HRM a partir de uma perspectiva internacional, concentrando-se mais nas economias desenvolvidas. Na importante contribuição sobre o tema, ainda vale destacar Bondarouk, Harms e Lepak (2017) que testaram empiricamente a importância da interação entre aspectos tecnológicos e organizacionais e a descoberta de que a HRM e a qualidade do serviço são consequências da implementação do e-HRM. Nesse sentido é observado que a e-HRM consta como proposta de impacto na GHRM (OBEIDAT, 2016; IQBAL et al., 2018).

Na percepção do potencial estratégico da e-HRM, Grant e Newell (2013) salientam o crescente corpo de trabalho que mostra que a gestão eficaz de RH pode fazer contribuições significativas para o desempenho organizacional e que as questões relacionadas a RH são centrais também para a criação de vantagem competitiva sustentável. Ainda referem que em razão do desenvolvimento organizacional contemporâneo, com o crescimento de organizações baseadas em conhecimento e em rede, a importância estratégica de questões relacionadas a RH provavelmente se tornará mais significativa no futuro.

$\mathrm{Na}$ teoria da troca social para explorar o papel da confiança impessoal como um fator intermediário de criação de valor entre a e-HRM e a produtividade, Iqbal, Ahmad e Allen (2019) propuseram buscar os antecedentes e as consequências da confiança impessoal nas organizações para fornecer uma visão holística da e-HRM e da produtividade dos trabalhadores. Sendo o primeiro estudo a examinar como a confiança impessoal medeia o relacionamento entre o e-HRM e a produtividade dos trabalhadores. Os resultados empíricos validaram todas as hipóteses do estudo, incluindo o papel da confiança impessoal, que medeia parcialmente a relação entre e-HRM e produtividade dos trabalhadores; ainda forneceram evidências empíricas de que a HRM habilitada pela tecnologia apoia as organizações, aprimorando a confiança organizacional e os resultados de produtividade.

Em uma abordagem configuracional, Galanaki, Lazazzara e Parry (2019) forneceram conhecimento sistemático sobre quais configurações de adoção de e-HRM realmente existem no nível global. Através da operacionalização da configuração de e-HRM como uma combinação do grau real de presença tecnológica e o grau em que a tecnologia é usada para permitir atividades de HRM. De acordo com as descobertas, quatro tipos de configuracões de 
“primado de TI". Além disso, o tamanho organizacional, o HRM e a concorrência nos mercados internacionais contribuem mais para determinar o tipo real de configuração do e-HRM. Os resultados sugerem também que o efeito das políticas nacionais que desencadeiam a inovação nas configurações de e-HRM deve ser considerado, em vez de clusters geográficos mais amplos.

A melhora no grau de inovação nas organizações foi investigada por Khashman (2019), sendo que o estudo resultou em cinco construtos de estratégias e-HRM: recrutamento eletrônico, a seleção eletrônica, o treinamento eletrônico, a avaliação do desempenho eletrônico e as compensações eletrônicas, e utilizou o papel mediador de um repositório de conhecimento para esses construtos para melhorar a inovação organizacional. Verificou-se que houve uma relação significativa forte e estatisticamente positiva entre várias estratégias e-HRM e inovação organizacional usando o repositório de conhecimento como um construto de mediação. Entretendo, trabalhadores e gestores que não fazem parte do $\mathrm{RH}$, apresentam resistência as práticas (BONDAROUK; RUEL, 2013), afirmando, inclusive, que não notaram mudanças perceptivas com a adoção de e-HRM na organização.

É comum que nas grandes organizações o uso de sistemas de informações para registrar, armazenar e analisar dados dos trabalhadores (BURBACH; ROYLE, 2014). Por meio de um modelo de difusão de e-HRM verificaram os fatores institucionais que determinam a difusão bem-sucedida de práticas de HRM em corporações multinacionais. Os resultados sugerem que a transferência bem-sucedida da e-HRM é mediada por um intercâmbio de vários fatores no nível institucional (externos, relacionais, organizacionais e individuais) dentro da corporação e de suas subsidiárias. A implementação bem-sucedida do e-HRM é sinônimo de integração e institucionalização bem-sucedidas das práticas de e-HRM nas subsidiárias.

Os resultados da pesquisa de Parry (2011), realizada em grande escala numa amostra de doze países, mostraram que o e-HRM pode ajudar o RH a aumentar seu valor tornando-se mais estratégico, mas não encontrou evidências de redução de custos devido a reduções no número de trabalhadores de RH. Isso sugere que as organizações estão usando o e-HRM para reimplantar os profissionais de RH do trabalho transacional para atividades mais estratégicas e de valor agregado. Mais adiante, investigando o alcance estratégico das organizações, Marley e Perry (2016) obtiveram que o envolvimento estratégico de RH e maior capacidade de e-HRM estão relacionados direta e reciprocamente, apoiando ambas as perspectivas teóricas, mas também mostrando que cada um não é mutuamente exclusivo.

Por meio de análises estatísticas Masum, Kabir e Chouwdhury (2015) verificaram que o suporte de gerenciamento de nível superior, os atributos individuais dos trabalhadores, a complexidade do sistema, a infraestrutura de TI e a pressão do setor são os determinantes mais influentes que influenciam a decisão de adoção do e-HRM. Competências intraempreendedoras podem possibilitar um maior engajamento e maior aderência a e-HRM. As descobertas revelam que os feitos e realizações das organizações de GHR com base em tecnologia não estão fora do alcance das instituições, no entanto, desempenho da competência e do engajamento dos trabalhadores (NURA; OSMAN, 2013).

Usar a abordagem de e-HRM para desenvolver a agilidade organizacional, Hamidianpour, Esmaeilpour e Firoozi (2016) buscaram respostas quantitativas para a questão. O modelo conceitual apresentado e indica que usando e-HRM a gestão pode afetar a agilidade organizacional significativamente Enquanto Bondarouk, Parry e Furtmueller revisaram quatro décadas de pesquisa nessa área, com o objetivo de fornecer uma estrutura resumida e integrativa como base para pesquisas futuras. Alguns fatores que afetam a adoção da GHR em linha podem ser divididos em três áreas: tecnologia, organização e pessoas. Divide-se as consequências em operacionais, relacionais e transformacionais. Para os outros houve uma mudança nas metas de GHR em linha, de eficiência para melhoria na prestação de serviçs: de RH R e na reorientação 
organização são essenciais para os processos inovativos, ou seja, o comportamento empreendedor torna-se uma competência necessária, como referem Baruah e Ward (2014).

Voltando-se um pouco para os resultados mais numéricos da revisão sistematizada, conforme pode ser observado na Tabela 2, da amostra selecionada, o primeiro artigo publicado foi em 2004 e a frequência de publicações apresenta crescimento, com o aumento na quantidade de artigos publicados nos últimos 6 anos, principalmente. Destaca-se o ano de 2013, ano que teve 7 publicações, significando $25 \%$ da amostra. Ainda, foi a partir desse ano que ocorreu uma alavancagem nas publicações, sendo que anteriormente, num espaço de oito anos (2004 - 2012), apenas 6 trabalhos científicos com o tema foram publicados.

Tabela 2 - Frequência de publicação por ano

\begin{tabular}{|c|c|c|}
\hline Ano de publicação & Quantidade & Frequência \% \\
\hline $\mathbf{2 0 0 4}$ & 1 & 3,6 \\
\hline $\mathbf{2 0 0 5}$ & 0 & 0 \\
\hline $\mathbf{2 0 0 6}$ & 0 & 0 \\
\hline $\mathbf{2 0 0 7}$ & 2 & 7,1 \\
\hline $\mathbf{2 0 0 8}$ & 0 & 0 \\
\hline $\mathbf{2 0 0 9}$ & 1 & 3,6 \\
\hline $\mathbf{2 0 1 0}$ & 0 & 0 \\
\hline $\mathbf{2 0 1 1}$ & 1 & 3,6 \\
\hline $\mathbf{2 0 1 2}$ & 1 & 3,6 \\
\hline $\mathbf{2 0 1 3}$ & 7 & 25,0 \\
\hline $\mathbf{2 0 1 4}$ & 1 & 3,6 \\
\hline $\mathbf{2 0 1 5}$ & 2 & 7,1 \\
\hline $\mathbf{2 0 1 6}$ & 5 & 17,9 \\
\hline $\mathbf{2 0 1 7}$ & 2 & 7,1 \\
\hline $\mathbf{2 0 1 8}$ & 1 & 3,6 \\
\hline $\mathbf{2 0 1 9}$ & 4 & 14,3 \\
\hline Total & 28 & 100 \\
\hline
\end{tabular}

Fonte: Dados da pesquisa (2019)

O autor com maior destaque na publicação de artigos sobre e-HRM é Tanya Bondarouk. Pode-se observar na Figura 1, nas linhas azuis, que são as publicações como primeiro autor e, em laranja, como segundo, Bondarouk destoa dos demais autores com seis publicações como primeira autora e 2 como segunda. Também se destaca Iqbal com 3 publicações como primeiro autor e, na sequência, Ruel com 2 publicações como primeiro autor e mais 3 como segundo autor. 


\section{Figura 1 - Autores com maior quantidade de publicação como primeiro e segundo autores}

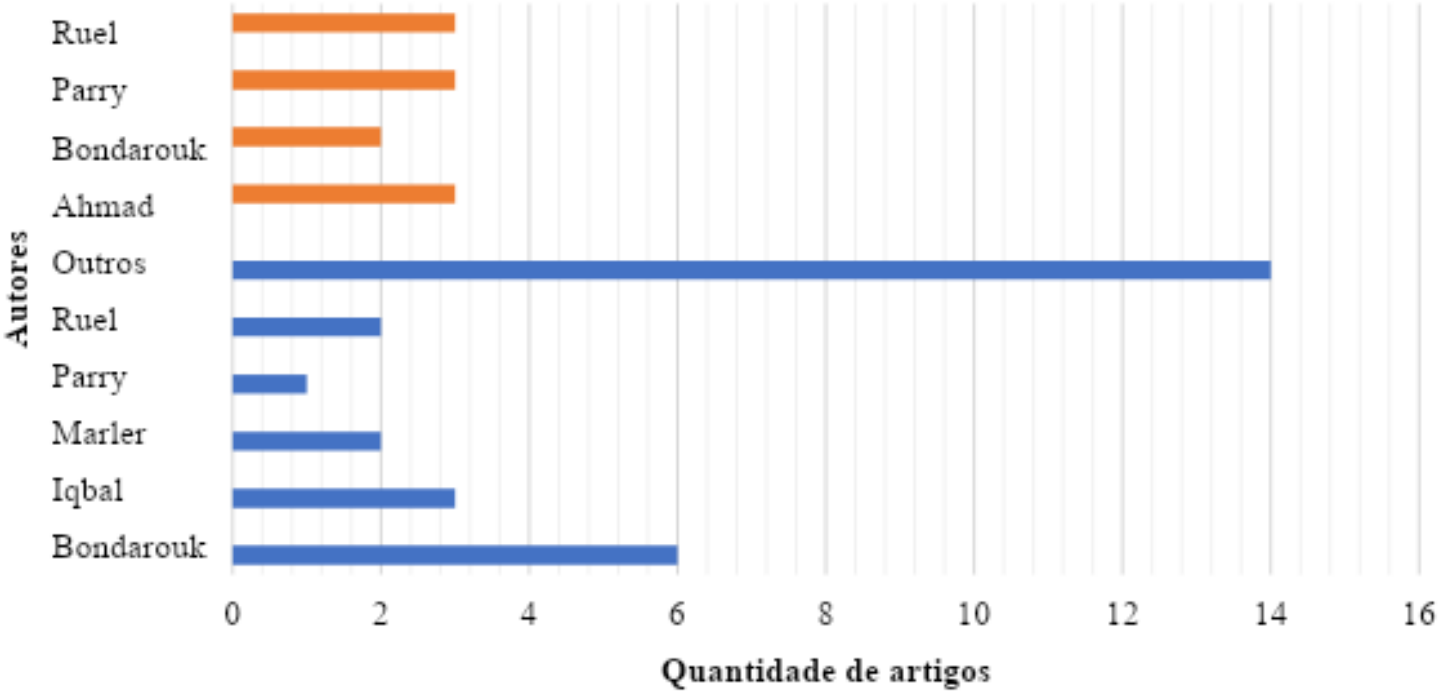

Fonte: Dados da pesquisa (2019)

Os dados da Figura 1 revela a concentração dos estudos em um número restrito de autores, os quais muitos se repetem em estudos de primeira autoria e segunda. Apesar de ser um tema emergente, e-HRM vem ganhando atenção dos pesquisadores e tende a ganhar ainda mais espaço, visto que, em síntese, o uso da estratégico da e-HRM nas organizações é justificado principalmente por auxiliar nos resultados organizacionais (PARRY, 2011; BAYARÇELIK; FINDIKLI, 2015; MARLER; PARRY, 2016; IQBAL et al., 2019). Tal aplicação tende a concentrar-se em práticas como: estabelecimento de metas, gerenciamento do tempo, a fácil aquisição e acesso a dados pessoais e a redução dos custos de administração, entre outras (BAYARÇELIK; FINDIKLI, 2015). O êxito organizacional está relacionado com a forma como as organizações gerem as pessoas que nelas trabalham (ESTEVES; CAETANO, 2010), portanto, as práticas de HRM influenciam nos resultados. E tudo isso vai ao encontro com trabalhadores intraempreendedores e a pressão da quarta revolução industrial.

\section{Considerações finais}

Chegando ao final desta revisão sistemática de literatura foi possível verificar que pressões institucionais criam tanto consequências transformacionais negativas que podem afetar substancialmente a capacidade de atingir o potencial estratégico da organização, quanto impactar no nível de desempenho dos trabalhadores (HEIKKILÄ, 2013). A HRM habilitada pela tecnologia (QBAL; AHMAD; ALLEN, 2019) apoia as organizações, aprimorando a confiança organizacional e os resultados de produtividade. Aponta-se a relação positiva entre variáveis estratégicas de e-HRM e inovação organizacional através de cinco construtos: recrutamento eletrônico, a seleção eletrônica, o treinamento eletrônico, a avaliação do desempenho eletrônico e as compensações eletrônicas (KHASHMAN, 2019).

No tocante ao desempenho organizacional, como no aumento da produtividade dos trabalhadores, a TI ajuda a modificar muitos processos de HRM, incluindo planejamento, recrutamento, seleção, gerenciamento de desempenho, fluxo de trabalho e remuneração. Além disso, na avaliação do desempenho dos trabalhadores individuais e uma definição clara dos resultados. Como afirmam Grant e Newell (2013) fica evidente o potencial estratégico da e-
Dol: 10.14211/xi-egepe-118087 
Assim sendo, pode se destacar a aplicação de e-HRM em diferentes organizações, de pequenos empreendimentos às organizações multinacionais. A implementação de e-HRM é sinônimo de integração e institucionalização entre práticas de e-HRM e trabalhadores. São notáveis as evidências de que importância estratégica de questões relacionadas a RH se tornará mais significativa no futuro. No entanto, conforme cresce a importância da tecnologia, cresce também a importância do capital humano. As competências intraempreendedoras são consideradas um diferencial entre os trabalhadores. Foi possível notar que a e-HRM é uma ferramenta eficiente, produtiva, inovadora e capaz de apoiar os objetivos estratégicos de uma organização, através de uma HRM estratégica.

Existem ainda muitas oportunidades para continuar refinando esse importante tema de pesquisa, como afirmam Marler e Fisher (2013), Bayarçelik e Findikli (2015) e Iqbal et al. (2019), inclusive, destaca-se a necessidade de mais estudos empíricos sobre e-HRM e resultados estratégicos de HRM em nível macro. Apesar do uso generalizado destes modelos, ocorre uma escassez surpreendente de teorias e pesquisas empíricas (STONE; DULEBOHN, 2013).

A finalidade desse estudo foi alcançada ao se verificar, por meio de uma revisão sistemática de literatura, que as práticas de e-HRM impactam nos resultados e no desempenho organizacional, sob a perspectiva do intraempreendedorismo. Fica evidenciado que as práticas de e-HRM dependem em grande parte das competências intraempreendedoras dos trabalhadores das organizações. Todavia, há muito a ser pesquisado nessa área, uma vez que é abrangente e o tema emergente. Como sugestões para estudos futuros apresenta-se a possibilidade de mais investigações no Brasil, sendo que são poucos os estudos encontrados sobre essa temática, constatando-se oportunidades tanto teóricas como empíricas, justamente para aprofundar os conhecimentos nesse tema também no contex to brasileiro. Nessa lógica, esta revisão fornece subsídios iniciais para pesquisadores interessados no tema.

\section{Referências}

ALMAZÁN, D. A.; TOVAR, Y. S.; QUINTERO, J. M. M. Influence of information systems on organizational results. Contaduría y Administración, v. 62, n. 2, p. 321-338, 2017.

ARMSTRONG, M. A handbook of human resource management practice. Kogan: Page Publishers, 2006.

ASH, S. R. Why should small businesses care about employee diversity?: Five areas of research that influence organizational results. Journal of Small Business \&

Entrepreneurship, v. 20, n. 3, p. 223-232, 2007.

BARNEY, J. Firm resources and sustained competitive advantage. Journal of management, v. 17, n. 1, p. 99-120, 1991.

BARUAH, B.; WARD, A. Metamorphosis of intrapreneurship as an effective organizational strategy. International Entrepreneurship and Management Journal, v.10, n.1, p.23-35, 2014.

BECKER, B. E.; Huselid, M. A. High performance work systems and firm performance: A synthesis of research and managerial implications. Research in personnel and human resource management. 1998 
BISSOLA, R.; IMPERATORI, B. Facing e-HRM: the consequences on employee attitude towards the organisation and the HR department in Italian SMEs. European Journal of International Management, v. 7, n. 4, p. 450-468, 2013.

BONDAROUK, T. V.; RUEL, H. Electronic Human Resource Management: challenges in the digital era. The International Journal of Human Resource Management, v. 20, n. 3, p. 505-514, 2009.

BONDAROUK, T.; RUEL, H. The strategic value of e-HRM: results from an exploratory study in a governmental organization. The International Journal of Human Resource Management, v. 24, n. 2, p. 391-414, 2013.

BONDAROUK, T.; BREWSTER, C. Conceptualising the future of HRM and technology research. The International Journal of Human Resource Management, v. 27, n. 21, p. 26522671, 2016.

BONDAROUK, T.; SCHILLING, D.; RUEL, H. e-HRM adoption in emerging economies: The case of subsidiaries of multinational corporations in Indonesia. Canadian Journal of Administrative Sciences/Revue Canadienne des Sciences de l'Administration, v. 33, n. 2, p. 124-137, 2016.

BONDAROUK, T.; HARMS, R.; LEPAK, D. Does e-HRM lead to better HRM service? The International Journal of Human Resource Management, v. 28, n. 9, p. 1332-1362, 2017.

BONDAROUK, T.; PARRY, E.; FURTMUELLER, E. Electronic HRM: four decades of research on adoption and consequences. The international Journal of human resource management. v. 28, n. 1, p. 98-131, 2017.

BURBACH, R.; ROYLE, T. Institutional determinants of e-HRM diffusion success. Employee Relations, v. 36, n. 4, p. 354-375, 2014.

COLTRE, J.; MARTINS, L. M. A industrial 4.0 na gestão estratégica: desafios e oportunidades para as empresas brasileiras. Revista Terra \& Cultura: Cadernos de Ensino e Pesquisa, v. 34, n. esp., p. 110-128, 2019.

CRONIN, P; RYAN, F.; COUGHLAN, M. Undertaking a literature review: a step-by-step approach. British journal of nursing, v. 17, n. 1, p. 38-43, 2008.

DELANEY, J. T.; HUSELID, M. A. The impact of human resource management practices on perceptions of org.anizational performance. Academy of Management journal, v. 39, n. 4, p. 949-969, 1996.

DRUCKER, P. A prática da gestão. Routledge, 2012.

ESTEVES, M. T.; CAETANO, A. Práticas de gestão de recursos humanos e resultados organizacionais: estudos, controvérsias teóricas e metodológicas. Revista Psicologia: Organizações e Trabalho, v. 10, n. 2, p. 159-176, 2010. 
FERRARESI, A. A.; SANTOS, S. A.; FREGA, J. R.; QUANDT, C. O. Os impactos da gestão do conhecimento na orientação estratégica, na inovatividade e nos resultados organizacionais: uma survey com empresas instaladas no Brasil. Revista de Administração Mackenzie (Mackenzie Management Review), v. 15, n. 2, 2014.

FILIPPI, A. C. G.; GUARNIERI, P.; CUNHA, C. A. Condomínios Rurais: revisão sistemática da literatura internacional. Estudos Sociedade e Agricultura, v. 27, n. 3, p. 525546, 2019.

FINDIKLI, M. A.; BAYARÇELIK, E. Exploring the outcomes of Electronic Human Resource Management (E-HRM)? Procedia-Social and Behavioral Sciences, v. 207, p. 424$431,2015$.

GALANAKI, E.; LAZAZZARA, A.; PARRY, E. A cross-national analysis of e-HRM configurations: integrating the information technology and HRM perspectives.

In: Organizing for Digital Innovation. Springer, Cham, p. 261-276, 2019.

GRANT, D.; NEWELL, S. Realizing the strategic potential of e-HRM. Journal of Strategic Information Systems, v. 3, n. 22, p. 187-192, 2013.

GRANT, R. M. Toward a knowledge-based theory of the firm. Strategic management journal, v. 17, n. S2, p. 109-122, 1996.

GUARNIERI, P. Síntese dos principais critérios, métodos e subproblemas da seleção de fornecedores multicritério. RAC-Revista de Administração Contemporânea, v. 19, n. 1, p. $1-25,2015$.

HAMIDIANPOUR, F.; ESMAEILPOUR, M.; FIROOZI, H. Assessing the Impact of Electronic Human Resource Management on Creation of Organizational Agility: A Study in the Bushehr Banks, Iran. Asian Social Science, v. 12, n. 7, 2016.

HASHIMOTO, M. O que é intraempreendedorismo.

2012. http://www.scielo.br/scielo.php?pid=S0103-0142017000200127\&script=sci_arttext, 2019.

HEIKKILÄ, J. An institutional theory perspective on e-HRM's strategic potential in MNC subsidiaries. The Journal of Strategic Information Systems, v. 22, n. 3, p. 238-251, 2013.

HIGGINS, J. P; GREEN, S. Cochrane handbook for systematic reviews of interventions. Version 5.1. The Cochrane Collaboration. www. cochrane-handbook. org, 2011.

IQBAL N.; AHMAD, M.; ALLEN, M. Unveiling the relationship between e-HRM, impersonal trust and employee productivity. Management Research Review, 2019.

IQBAL, N.; AHMAD, M.; RAZIQ, M. M.; BORINI, F. M. Linking e-hrm practices and organizational outcomes: empirical analysis of line manager's perception. Revista Brasileira de Gestão de Negócios, v. 21, n. 1, p. 48-69, 2019. 
IQBAL, N.; AHMAD, M.; ALLEN M.; RAZIQ, M. M. Does e-HRM improve labour productivity? A study of commercial bank workplaces in Pakistan. Employee Relations, v. 40, n. 2, p. 281-297, 2018.

ISLAMI, X.; MULOLLI, E.; MUSTAFA, N. Using Management by Objectives as a performance appraisal tool for employee satisfaction. Future Business Journal, v. 4, n. 1, p. 94-108, 2018.

KHASHMAN, A. M. The Impact of Electronic Human Resource Management (E-HRM) Strategies on Organizational Innovation by Knowledge Repository as Mediating Role. International Journal of Web Portals (IJWP), v. 11, n. 1, p. 19-38, 2019.

LAPOLLI, É; GOMES, R. K. Práticas intraempreendedoras na gestão pública: um estudo de caso na Embrapa. Estudos Avançados, v. 31, n. 90, p. 127-142, 2017.

Management, v. 7, n. 4, p. 450-468, 2013.

MARLER, J. H.; FISHER, S. L. An evidence-based review of e-HRM and strategic human resource management. Human Resource Management Review, v. 23, n. 1, p. 18-36, 2013.

MARLER, J. H.; PARRY, E. Human resource management, strategic involvement and eHRM technology. The International Journal of Human Resource Management, v. 27, n. 19, p. 2233-2253, 2016.

MARTINS, E. R.; Geraldes, W. B.; Afonseca, U. R.; Gouveia, L. M. B. Tecnologias Móveis em Contexto Educativo: uma Revisão Sistemática da Literatura. RENOTE, v. 16, n. 1, 2018.

MASUM, A. K. M; KABIR, M. J.; CHOWDHURY, M. M. Determinants that influencing the adoption of E-HRM: An empirical study on Bangladesh. Asian Social Science, v. 11, n. 21, p. 117, 2015.

MUNHOZ, C. E.; NASSIF, V. M. J. O desvelar das competências dos intraempreendedores: um estudo exploratório em empresas de serviços. Revista Economia \& Gestão, v. 12, n. 30, p. 20-48, 2012.

NASSIF, V. M. J.; ANDREASSI, T.; SIMÕES, F. Competências empreendedoras: há diferenças entre empreendedores e intraempreendedores? RAI Revista de Administração e Inovação, v. 8, n. 3, p. 33-54, 2011.

NURA, A. A.; OSMAN, N. H. Gauging the effect of performance management and technology based human resource management on employee retention: The perspective of academics in higher educational institutions in Sokoto State Nigeria. Asian Social Science, v. 9, n. 15, p. 295, 2013.

OBEIDAT, S. M. The link between e-HRM use and HRM effectiveness: an empirical study. Personnel review, v. 45, n. 6, p. 1281-1301, 2016.

PARRY, E. An examination of e-HRM as a means to increase the value of the HR

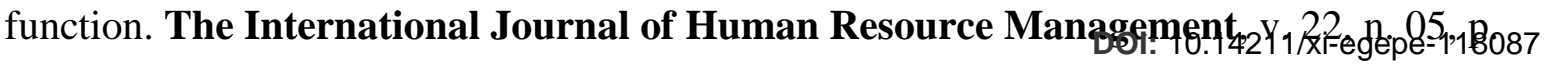


PENROSE, E. The theory ofthe growth ofthe firm. JohnW iley\& Sons, New York, 1959.

PEREIRA, L. M.; HASHIMOTO, M. Das razões que levam funcionários de empresas intraempreendedoras a constituírem seus próprios negócios. Encontro de Estudos em Empreendedorismo e Gestão de Pequenas Empresas, v. 8, 2014.

PETERAF, M. A. The cornerstones of competitive advantage: a resource-based view. Strategic management journal, v. 14, n. 3, p. 179-191, 1993.

PINCHOT, G. III. Intrapreneuring: porque você não precisa deixar a empresa para tornarse um empreendedor. Trad. Nivaldo Montingelli Jr. São Paulo: Harbra, 1989.

RUEL, H.; BONDAROUK, T.; VAN DER VELDE, M. The contribution of e-HRM to HRM effectiveness: Results from a quantitative study in a Dutch Ministry. Employee relations, v. 29, n. 3, p. 280-291, 2007.

RUEL, H.; BONDAROUK, T.; LOOISE, J. E-HRM: Innovation or irritation. An explorative empirical study in five large companies on web-based HRM. Management revue, p. 364380, 2004.

SAMPAIO, R. F.; MANCINI, M. C. Estudos de revisão sistemática: um guia para síntese criteriosa da evidência científica. Rev. Bras. de fisioterapia. v.11, n. 1, p. 83-89, 2007.

STONE, D. L.; DULEBOHN, J. H. Emerging issues in theory and research on electronic human resource management (eHRM). Human Resource Management Review. v. 23, n.1, p.1-5, 2013.

STROHMEIER, S. R. e-HRM: Review and implications. Human resource management review, v. 17, n. 1, p. 19-37, 2007.

VENKATRAMAN, N.; RAMANUJAM, V. Measurement of business economic performance: An examination of method convergence. Journal of management, v. 13, n. 1, p. 109-122, 1987.

WERNERFELT, B. A resource-based view of the firm. Strategic management journal, v. 5, n. 2, p. 171-180, 1984.

YIN, R. K. Pesquisa qualitativa do início ao fim. Porto Alegre: Penso, 2016.

YUSLIZA, M. Y.; RAMAYAH, T. Determinants of attitude towards E-HRM: an empirical study among HR professionals. Procedia-Social and Behavioral Sciences, v. 57, p. 312-319, 2012. 